\title{
Hydronium-dominated ion transport in carbon-dioxide-saturated electrolytes at low salt concentrations in nanochannels
}

Lund Jensen, Kristian; Kristensen, Jesper Toft; Crumrine, Andrew Michael; Andersen, Mathias Bækbo; Bruus, Henrik; Pennathur, Sumita

Published in:

Physical Review E

Link to article, DOI:

10.1103/PhysRevE.83.056307

Publication date:

2011

Document Version

Publisher's PDF, also known as Version of record

Link back to DTU Orbit

Citation $(A P A)$ :

Lund Jensen, K., Kristensen, J. T., Crumrine, A. M., Andersen, M. B., Bruus, H., \& Pennathur, S. (2011). Hydronium-dominated ion transport in carbon-dioxide-saturated electrolytes at low salt concentrations in nanochannels. Physical Review E, 83(5), artikle number 056307. https://doi.org/10.1103/PhysRevE.83.056307

\section{General rights}

Copyright and moral rights for the publications made accessible in the public portal are retained by the authors and/or other copyright owners and it is a condition of accessing publications that users recognise and abide by the legal requirements associated with these rights.

- Users may download and print one copy of any publication from the public portal for the purpose of private study or research.

- You may not further distribute the material or use it for any profit-making activity or commercial gain

- You may freely distribute the URL identifying the publication in the public portal 


\title{
Hydronium-dominated ion transport in carbon-dioxide-saturated electrolytes at low salt concentrations in nanochannels
}

\author{
Kristian Lund Jensen, ${ }^{1}$ Jesper Toft Kristensen, ${ }^{1}$ Andrew Michael Crumrine, ${ }^{2}$ Mathias Bækbo Andersen, ${ }^{1}$ \\ Henrik Bruus, ${ }^{1}$ and Sumita Pennathur ${ }^{2}$ \\ ${ }^{1}$ Department of Micro- and Nanotechnology, Technical University of Denmark, \\ DTU Nanotech Building 345 East, DK-2800 Kongens Lyngby, Denmark \\ ${ }^{2}$ Department of Mechanical Engineering, University of California, Santa Barbara, California 93106, USA \\ (Received 19 August 2010; revised manuscript received 19 January 2011; published 9 May 2011)
}

\begin{abstract}
Nanochannel ion transport is known to be governed by surface charge at low ionic concentrations. In this paper, we show that this surface charge is typically dominated by hydronium ions arising from dissolution of ambient atmospheric carbon dioxide. Taking the hydronium ions into account, we model the nanochannel conductance at low salt concentrations and identify a conductance minimum before saturation at a value independent of salt concentration in the dilute limit. Via the Poisson-Boltzmann equation, our model self-consistently couples chemical-equilibrium dissociation models of the silica wall and of the electrolyte bulk, parametrized by the dissociation reaction constants. Experimental data with aqueous $\mathrm{KCl}$ solutions in 165-nm-high silica nanochannels are described well by our model, both with and without extra hydronium from added $\mathrm{HCl}$.
\end{abstract}

PACS number(s): 47.57.jd, 47.61.-k, 66.10.-x, 82.65.+r

\section{INTRODUCTION}

Nanofluidics and specifically, ion transport through artificial nanochannels, are important for both fundamental scientific studies and for practical biomolecular and energybased applications [1-3]. Of particular interest is the intricate interplay among surface chemistry, electrokinetics, and fluid dynamics spanning over molecular and continuum macroscopic length scales [4,5]. It has been demonstrated that the electrokinetic properties at this scale have enabled a range of innovations including those for chemical sensing and bioanalytics [6-12], energy harvesting systems, [13-18], and nanofluidic ion transport [19-26], including enrichment, depletion, and rectification effects [27-32].

For many of these applications, characterizing and understanding the conduction properties of electrolyte-filled nanochannels at low salt concentrations $\left(<10^{-3} \mathrm{M}\right)$ is important, and this raises the question of the role of hydronium ions. It is well known that, under standard conditions, the dissolution of ambient atmospheric carbon dioxide in de-ionized (DI) water leads to the formation of carbonic acid, which, upon reaching chemical equilibrium with the water, gives rise to an inherent concentration of hydronium ions of $\sim 10^{-6} \mathrm{M}$ corresponding to $\mathrm{pH}$ 5.7. Because hydronium has a uniquely high mobility, a factor of $\sim 5$ higher than common salt ions, in bulk solutions, it is found that hydronium ions begin to dominate the electrical conductivity when the salt concentration is lower than $\sim 5 \times 10^{-6} \mathrm{M}$. Furthermore, hydronium is also known to interact with the confining walls of the electrolyte. For oxide walls, most prominently silica, numerous studies have shown how hydronium affects the electrical properties of the wall-electrolyte interface and leads to a wall surface charge that depends on salt concentration [33-44] including our own recent study [26]. Finally, at sufficiently low salt concentration, this surface charge is found to dominate the conductance of electrolyte-filled nanochannels [8,11,19,24,27,28,32,45]. Given these facts, it is remarkable that the role of hydronium ions is largely unexplored in the literature on nanochannel conductance at low salt concentrations in unbuffered solutions.
TABLE I. Experimental observations of a nanochannel conductance minimum at low salt concentration: List of reference, figure number, year, magnitude $\delta$ of the minimum Eq. (21), and the applied surface-charge model (Const and Dissoc refer to constant surfacecharge and chemical-equilibrium dissociation models, respectively). All studies involve silica nanochannels and aqueous $\mathrm{KCl}$ solutions, except Stein et al., who used a 50\%/50\% mixture of isopropanol and an appropriately diluted $\mathrm{KCl}$ : TRIS $(100: 1)$ solution.

\begin{tabular}{lccccc}
\hline \hline References & Figure & Year & $\delta$ & Model \\
\hline$[19]$ & Stein et al. & 3 & $(2004)$ & 1.06 & Const \\
{$[28]$} & Karnik et al. & 3 & $(2005)$ & 1.38 & Const \\
{$[45]$} & Schoch and Renaud & 3 & $(2005)$ & 1.19 & Const \\
{$[46]$} & Cheng & 2.9 & $(2008)$ & 1.27 & Const \\
{$[24]$} & Martins et al. & 3 & $(2009)$ & 1.68 & Const \\
{$[32]$} & Duan et al. & 3 & $(2010)$ & 1.25 & Dissoc \\
& This paper & - & $(2010)$ & 1.18 & Dissoc \\
\hline \hline
\end{tabular}

The main goal of this paper is to provide such an analysis mainly in terms of theoretical modeling, but also supported by our own experimental validation. Among the dozens of papers published on nanochannel conductance, we have only found a single very recent paper dealing directly with similar modeling [32].

Another motivation for our paper is to explain the appearance of a minimum in the nanochannel conductance as a function of salt concentration, which several groups have observed, but not noticed, see Table I. The minimum, which cannot be explained by depletion effects near the entrances, as this would lead to a decreased conductance at the lowest salt concentration, is found to occur for salt concentrations $\sim 5 \times 10^{-6} \mathrm{M}$, the above-mentioned crossover from salt to hydronium-dominated conductance. The main result of our analysis is that our model predicts such a minimum due to the presence of inherent hydronium ions. 


\section{MODEL}

We consider a long straight nanochannel connecting two large bulk reservoirs. Traditionally, theoretical modeling of ionic transport in such systems is based on the GouyChapman-Stern theory of electrostatic screening by the mobile co- and counterions in an aqueous salt solution of a wall with constant surface charge, coupled to the continuum fluid dynamics equations and, thus, forming the PoissonNernst-Planck transport equations [47]. This results in a monotonic decreasing nanochannel conductance as a function of decreasing salt concentration that levels off at a plateau in the dilute limit, as shown in Refs. [19,24,28,45,46] of Table I as well as in Refs. $[8,11,27]$ and summarized by Fig. 8 in a recent review paper [2]. However, this is in contrast to the observed nonmonotonic conductance graphs with a minimum, and therefore, we choose to base our analysis on the other well-known class of modeling, where the surface charge is governed dynamically by chemical reaction constants of the proton dissociation processes in the bulk electrolyte and at the wall [26,32-44].

Building on our own recently published work [26], we extend these previous buffer/wall dissociation models to low concentration unbuffered electrolyte systems by the addition of two crucial features: First, we account for hydronium ions (here denoted as $\mathrm{H}^{+}$for brevity) from autoprotolysis of water and from dissociated carbonic acid induced by dissolution of ambient atmospheric carbon dioxide; and second, we calculate the surface charge from a self-consistent electrostatic coupling between hydronium dissociation models of the silanol groups at the wall and of all the bulk constituents, parametrized by the associated dissociation constants. Our model consists of three parts: (i) bulk dissociation reactions determining the concentrations of the involved $\mathrm{H}^{+}, \mathrm{OH}^{-}, \mathrm{HCO}_{3}^{-}, \mathrm{CO}_{3}^{2-}$, $\mathrm{K}^{+}$, and $\mathrm{Cl}^{-}$ions in the reservoirs, (ii) surface reactions determining the potential and charge of the nanochannel surface, and (iii) the Poisson-Boltzmann equation for the electrical potential coupling the first two parts.

Specifically, following Refs. [26,33-44], we model the solid/liquid interface as the layered structure shown in Fig. 1. The innermost plane is the silanol surface at the o plane with surface charge $\sigma_{\mathrm{o}}$ and potential $\phi_{\mathrm{o}}$. Next is the immobile Stern layer situated between the o plane and the $\mathrm{d}$ plane and having the capacitance $C_{\mathrm{s}}\left[\mathrm{F} / \mathrm{m}^{2}\right]$. Following the Stern layer is the electrically charged diffusive screening layer extending from the $d$ plane a few times the Debye screening length $\lambda_{D}$ to the bulk and having surface charge $\sigma_{\mathrm{d}}$ and $\zeta$ potential $\phi_{\mathrm{d}}$. The last layer is the charge-neutral bulk of the reservoirs.

\section{A. Bulk chemistry of the reservoirs}

All bulk ionic concentrations in the reservoirs are calculated using the method of chemical families presented in Ref. [48] and supplemented by an open-source MATLAB-code buffer calculator [49]. For this paper, the carbonic acid family $\mathrm{H}_{2} \mathrm{CO}_{3}$ with charge states $z=0,-1$, and -2 is of particular interest,

$$
\begin{aligned}
\mathrm{H}_{2} \mathrm{CO}_{3} & \rightleftharpoons \mathrm{HCO}_{3}^{-}+\mathrm{H}^{+}, \quad \mathrm{p} K_{\mathrm{HCO}_{3}^{-}}=6.35 \\
\mathrm{HCO}_{3}^{-} & \rightleftharpoons \mathrm{CO}_{3}^{2-}+\mathrm{H}^{+}, \quad \mathrm{p} K_{\mathrm{CO}_{3}^{2-}}=10.33
\end{aligned}
$$

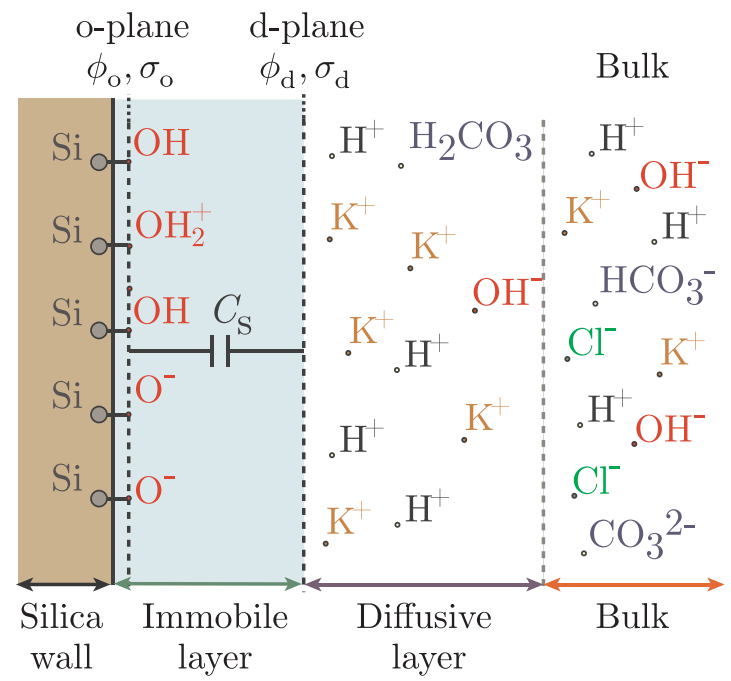

FIG. 1. (Color online) Model of a silica surface (dark brown) contacting an aqueous solution of $\mathrm{KCl}$ (white). At the bottom, the four regions of our model have been identified: the silica wall, the immobile Stern layer (light blue), the diffusive layer, and the bulk. The dashed line denoted as the o plane is where the bound surface charge $\sigma_{\mathrm{o}}$ resides, while the dashed line denoted as the d plane marks the beginning of the diffuse mobile layer, a layer stretching from the $\mathrm{d}$ plane to the bulk, and in which a mobile screening charge $\sigma_{\mathrm{d}}=-\sigma_{\mathrm{o}}$ resides. $C_{\mathrm{s}}$ is the (Stern) capacitance of the immobile layer. The potentials at the o and d planes are denoted as $\phi_{\mathrm{o}}$ and $\phi_{\mathrm{d}}$, respectively.

For DI water with a saturated concentration of carbonic acid of $11.8 \mu \mathrm{M}$ at $25^{\circ} \mathrm{C}$ [50], $\mathrm{pH}=5.68$, in agreement with our own experimental measurements. Likewise, the salt $\mathrm{KCl}$ is represented by the potassium hydroxide family $\mathrm{KOH}$, with charge states $z=0$ and +1 , together with the hydrochloric acid family $\mathrm{HCl}$ with charge states $z=0$ and -1 . A list of all involved chemical families taken into account in our model is shown in Table II.

When the total concentration $c_{\mathrm{X}}^{\text {tot }}$ of each chemical family in a given aqueous solution is known, the equilibrium reactions listed in Table II can be solved with respect to the bulk concentration $c_{\mathrm{X}, z \mathrm{X}}^{\mathrm{b}}$ of each family member using the buffer calculator code [49]. This calculational scheme takes dissociation equilibrium, conservation of mass, and charge neutrality into account,

$$
\begin{aligned}
K_{\mathrm{X}, z_{\mathrm{X}}} c_{\mathrm{X}, z_{\mathrm{X}}+1}^{\mathrm{b}} & =c_{\mathrm{X}, z_{\mathrm{X}}}^{\mathrm{b}} c_{\mathrm{H}}^{\mathrm{b}}, \text { dissociation reactions, } \\
\sum_{z_{\mathrm{X}}} c_{\mathrm{X}, z_{\mathrm{X}}}^{\mathrm{b}} & =c_{\mathrm{X}}^{\mathrm{tot}}, \text { conservation of mass, } \\
\sum_{\mathrm{X}, z_{\mathrm{X}}} z_{\mathrm{X}} c_{\mathrm{X}, z_{\mathrm{X}}}^{\mathrm{b}} & =0, \text { charge neutrality. }
\end{aligned}
$$

As Eqs. (2) describe the bulk ionic composition in the reservoirs, charge neutrality is a good approximation.

Once the bulk concentrations $c_{\mathrm{X}, z_{\mathrm{X}}}^{\mathrm{b}}$ are known, three parameters characterizing the bulk electrolyte can be determined: the 
TABLE II. List of the chemical families $X$ used in this paper together with the associated reaction schemes, charge states $z_{\mathrm{X}}$, reaction constants $\mathrm{p} K_{\mathrm{X}, z_{\mathrm{X}}}=-\log _{10}\left(K_{\mathrm{X}, z_{\mathrm{X}}} / 1 \mathrm{M}\right)$, and electrophoretic mobilities $\mu_{\mathrm{X}, z_{\mathrm{X}}}^{\mathrm{b}}$. Note that the $\mathrm{p} K_{\mathrm{X}, z_{\mathrm{X}}}$ values are always associated with dissociation processes.

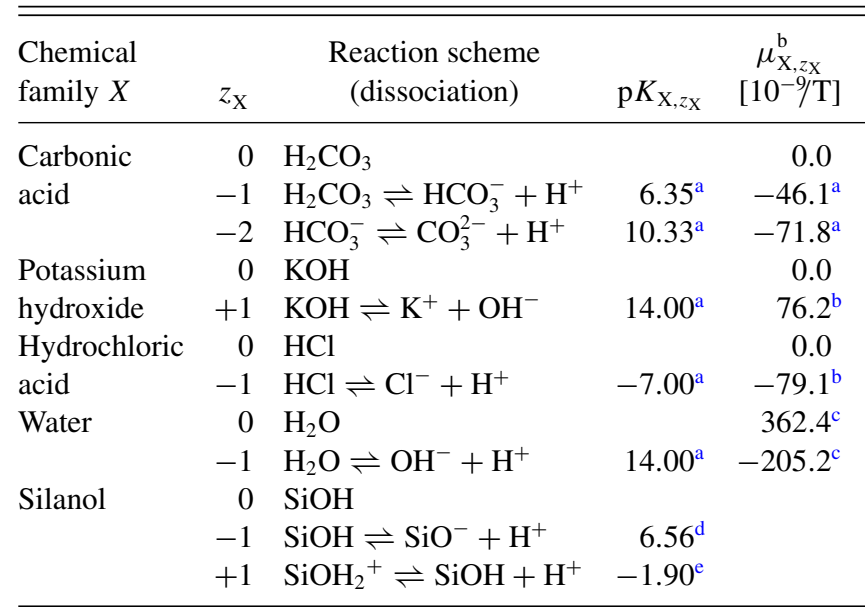

${ }^{\mathrm{a}}$ From Ref. [52], infinite dilution at $25^{\circ} \mathrm{C}$.

${ }^{\mathrm{b}}$ From Ref. [53], infinite dilution at $25^{\circ} \mathrm{C}$.

${ }^{\mathrm{c}}$ Here, $z=0$ represents $\mathrm{H}^{+}$, and $z=-1$ represents $\mathrm{OH}^{-}$.

${ }^{\mathrm{d}}$ From this paper.

${ }^{\mathrm{e}}$ From Ref. [38].

ionic strength $c_{I}$, the dilute-limit electric conductivity $\sigma_{\mathrm{el}}^{\mathrm{o}}$, and the Debye screening length $\lambda_{\mathrm{D}}$,

$$
\begin{aligned}
c_{I} & =\frac{1}{2} \sum_{\mathrm{X}, z_{\mathrm{X}}} z_{\mathrm{X}}^{2} c_{\mathrm{X}, z_{\mathrm{X}}}^{\mathrm{b}}, \\
\sigma_{\mathrm{el}}^{\mathrm{o}} & =e \sum_{\mathrm{X}, z_{\mathrm{X}}} z_{\mathrm{X}} \mu_{\mathrm{X}, z_{\mathrm{X}}}^{\mathrm{b}} c_{\mathrm{X}, z \mathrm{X}}^{\mathrm{b}}, \\
\lambda_{\mathrm{D}} & =\left[\frac{\epsilon k_{\mathrm{B}} T}{2 e^{2} c_{I}}\right]^{1 / 2} .
\end{aligned}
$$

At ionic strengths above $1 \mathrm{mM}$, the dilute-limit electrophoretic mobility $\mu_{\mathrm{X}, z_{\mathrm{X}}}^{\mathrm{b}}$ in Eq. (3b) is inaccurate, and we correct it using the Pitts equation, which, for a $1: 1$ electrolyte in water at $25^{\circ} \mathrm{C}$, takes the form [52]

$$
\mu_{\mathrm{X}, z_{\mathrm{X}}}^{* \mathrm{~b}}=\mu_{\mathrm{X}, z_{\mathrm{X}}}^{\mathrm{b}}-\left(A z_{\mathrm{X}}+0.23\left|z_{\mathrm{X}} z_{\mathrm{X}, \mathrm{ci}}\right| \mu_{\mathrm{X}, z_{\mathrm{X}}}^{\mathrm{b}}\right) \frac{\sqrt{c_{I}}}{1+a B \sqrt{c_{I}}},
$$

where $\mu_{\mathrm{X}, z_{\mathrm{X}}}^{* \mathrm{~b}}$ is the mobility corrected for nonzero ionic strength, $A=3.1 \times 10^{-8} \mathrm{~m}^{2} \mathrm{~V}^{-1} \mathrm{~s}^{-1}$ is a constant, $z_{\mathrm{X}, \mathrm{ci}}$ is the valence of the counterion, $B=0.33 \AA^{-1} \mathrm{M}^{-1 / 2}$ is a constant, and $a$ is an effective atomic radius [52]. In this paper, the conductivity in the high ionic strength regime is dominated by $\mathrm{K}^{+}$and $\mathrm{Cl}^{-}$ions, for which $a=3 \AA$ [54], and therefore, we approximate the electrolyte as binary. We can then use these two corrected mobilities for $\mathrm{K}^{+}$and $\mathrm{Cl}^{-}$in Eq. (3b) to improve the accuracy of our calculated electrical conductivity in the high ionic strength regime.

\section{B. Nanochannel electrostatics}

Near the walls in any given electrolyte system, charge transfer processes occur between the electrolyte and the wall, leading to a nonzero electric potential $\phi(\boldsymbol{r})$. Defining $\phi$ to be zero in the bulk reservoirs, and assuming that the ionic concentrations are small enough to neglect interionic correlations, the concentration $c_{\mathrm{X}, z_{\mathrm{X}}}$ of the ions as a function of position $\boldsymbol{r}$ in charge state $z_{X}$ of family $X$ can be written in terms of the bulk concentration $c_{\mathrm{X}, z_{\mathrm{X}}}^{\mathrm{b}}$ multiplied by a Boltzmann distribution factor as

$$
c_{\mathrm{X}, z_{\mathrm{X}}}(\boldsymbol{r})=c_{\mathrm{X}, z_{\mathrm{X}}}^{\mathrm{b}} \exp \left[-\frac{z_{\mathrm{X}} e \phi(\boldsymbol{r})}{k_{\mathrm{B}} T}\right],
$$

where we take the electric potential to be zero in the reservoirs. It simplifies the analysis if we nondimensionalize electric potentials and concentrations by introducing $k_{\mathrm{B}} T / e$ as the scale for the electric potentials and $c_{I}$ as the scale for the concentrations. The dimensionless fields, denoted by a tilde, become

$$
\tilde{\phi}=\frac{e}{k_{\mathrm{B}} T} \phi, \quad \tilde{c}_{\mathrm{X}, z \mathrm{X}}=\frac{1}{c_{I}} c_{\mathrm{X}, z_{\mathrm{X}}} .
$$

Henceforth, the electric charge density can be written as $\rho_{\mathrm{el}}=e c_{I} \sum_{\mathrm{X}, z_{\mathrm{X}}} z_{\mathrm{X}} \tilde{c}_{\mathrm{X}, z \mathrm{X}}$, which together with Eq. (5) leads to the Poisson-Boltzmann equation for the dimensionless potential $\tilde{\phi}$,

$$
\nabla^{2} \tilde{\phi}=-\frac{e \rho_{\mathrm{el}}}{\epsilon k_{\mathrm{B}} T}=-\frac{1}{2 \lambda_{\mathrm{D}}^{2}} \sum_{\mathrm{X}, z_{\mathrm{X}}} z_{\mathrm{X}} \tilde{c}_{\mathrm{X}, z_{\mathrm{X}}}^{\mathrm{b}} e^{-z_{\mathrm{X}} \tilde{\phi}} .
$$

For a symmetric binary electrolyte, this reduces to the wellknown simple form $\nabla^{2} \tilde{\phi}=\sinh (\tilde{\phi}) / \lambda_{\mathrm{D}}^{2}$.

\section{Nanochannel surface chemistry}

The total surface density of charge-active silanol sites at the wall is denoted as $\Gamma_{\text {tot }}\left[\mathrm{m}^{-2}\right]$. In our model, we take the three charge states $z=0,-1$, and +1 into account, for $\mathrm{SiOH}, \mathrm{SiO}^{-}$, and $\mathrm{SiOH}_{2}{ }^{+}$, respectively, as well as the two associated hydronium dissociation processes listed under silanol in Table II. These surface charge states have the densities $\Gamma_{o}, \Gamma_{-}$, and $\Gamma_{+}$, respectively, and it is natural to nondimensionalize them relative to $\Gamma_{\text {tot }}$ to obtain

$$
\tilde{\Gamma}_{\mathrm{o}}=\frac{1}{\Gamma_{\text {tot }}} \Gamma_{\mathrm{o}}, \quad \tilde{\Gamma}_{-}=\frac{1}{\Gamma_{\mathrm{tot}}} \Gamma_{-}, \quad \tilde{\Gamma}_{+}=\frac{1}{\Gamma_{\mathrm{tot}}} \Gamma_{+},
$$

with the obvious normalization condition

$$
\tilde{\Gamma}_{\mathrm{o}}+\tilde{\Gamma}_{-}+\tilde{\Gamma}_{+}=1
$$

The hydronium dissociation processes at the wall involve the concentration $c_{\mathrm{H}}^{\mathrm{o}}$ of the hydronium ions at the o plane, which, through the Boltzmann distribution, is related to the bulk concentration $c_{\mathrm{H}}^{\mathrm{b}}$. Consequently, the dimensional dissociation constants $K_{ \pm}$for the two surface processes as well as their nondimensionalized counterparts $\tilde{K}_{ \pm}=K_{ \pm} / c_{\mathrm{H}}^{\mathrm{b}}$ become

$$
\begin{aligned}
K_{-}=\frac{\Gamma_{-}}{\Gamma_{\mathrm{o}}} c_{\mathrm{H}}^{\mathrm{b}} e^{-\left(e / k_{\mathrm{B}} T\right) \phi_{\mathrm{o}}}, & \tilde{K}_{-}=\frac{\tilde{\Gamma}_{-}}{\tilde{\Gamma}_{\mathrm{o}}} e^{-\tilde{\phi}_{\mathrm{o}}}, \\
K_{+}=\frac{\Gamma_{\mathrm{o}}}{\Gamma_{+}} c_{\mathrm{H}}^{\mathrm{b}} e^{-\left(e / k_{\mathrm{B}} T\right) \phi_{\mathrm{o}}}, & \tilde{K}_{+}=\frac{\tilde{\Gamma}_{\mathrm{o}}}{\tilde{\Gamma}_{+}} e^{-\tilde{\phi}_{\mathrm{o}}} .
\end{aligned}
$$


The physical $\mathrm{p} K$ values $\mathrm{p} K_{ \pm}=-\log _{10}\left(K_{ \pm} / 1 \mathrm{M}\right)$ are listed in Table II. The surface charge $\sigma_{\mathrm{o}}$ and its nondimensionalized counterpart $\tilde{\sigma}_{\mathrm{o}}$ normalized by $e \Gamma_{\text {tot }}$ are

$$
\sigma_{\mathrm{o}}=e\left(\Gamma_{+}-\Gamma_{-}\right), \quad \tilde{\sigma}_{\mathrm{o}}=\tilde{\Gamma}_{+}-\tilde{\Gamma}_{-} .
$$

Finally, by straightforward algebra combining Eqs. (9)-(11), we eliminate $\tilde{\Gamma}_{\mathrm{o}}, \tilde{\Gamma}_{+}$, and $\tilde{\Gamma}_{-}$and obtain an expression of $\tilde{\sigma}_{\mathrm{o}}$ in terms of $\tilde{\phi}_{\mathrm{o}}$ and $\tilde{K}_{ \pm}$,

$$
\tilde{\sigma}_{\mathrm{o}}\left(\tilde{\phi}_{\mathrm{o}}\right)=\frac{e^{-2 \tilde{\phi}_{\mathrm{o}}}-\tilde{K}_{+} \tilde{K}_{-}}{e^{-2 \tilde{\phi}_{\mathrm{o}}}+\tilde{K}_{+} e^{-\tilde{\phi}_{\mathrm{o}}}+\tilde{K}_{+} \tilde{K}_{-}} .
$$

\section{Relating the surface to the bulk}

The above surface and bulk ionic concentrations can be coupled by the Poisson-Boltzmann equation. Whereas, hydronium ions can diffuse to the o plane, other ions may not penetrate past the $\mathrm{d}$ plane, forming the immobile Stern layer depicted in Fig. 1.

Following the Gouy-Chapman-Stern model, we introduce the Stern capacitance $C_{\mathrm{s}}\left[\mathrm{F} / \mathrm{m}^{2}\right]$ (nondimensionalized as $\tilde{C}_{\mathrm{s}}$ ). It enters in an assumed linear relation between the voltage drop $\phi_{\mathrm{o}}-\phi_{\mathrm{d}}$ across the immobile layer and the surface charge $\sigma_{\mathrm{o}}$,

$$
\phi_{\mathrm{o}}-\phi_{\mathrm{d}}=\frac{\sigma_{\mathrm{o}}}{C_{\mathrm{s}}}, \quad \tilde{\phi}_{\mathrm{o}}-\tilde{\phi}_{\mathrm{d}}=\frac{\tilde{\sigma}_{\mathrm{o}}}{\tilde{C}_{\mathrm{s}}}, \quad \tilde{C}_{\mathrm{s}}=\frac{k_{\mathrm{B}} T}{e^{2} \Gamma_{\mathrm{tot}}} C_{\mathrm{s}} .
$$

The boundary conditions at the surface for the PoissonBoltzmann equation (7) involve the surface charge $\tilde{\sigma}_{0}$ through Eqs. (12) and (13), and they become both nonlinear and mixed,

$$
\begin{gathered}
\boldsymbol{n} \cdot \nabla \tilde{\phi}=\frac{1}{\lambda_{\mathrm{D}} \tilde{q}_{I}} \tilde{\sigma}_{\mathrm{o}}(\tilde{\phi}), \text { at the d plane, } \\
\tilde{q}_{I}=\frac{\sqrt{2 \epsilon k_{\mathrm{B}} T c_{I}}}{e \Gamma_{\text {tot }}} .
\end{gathered}
$$

Here, we have introduced the surface normal $\boldsymbol{n}$ and the nondimensionalized bulk charge area density $\tilde{q}_{I}$.

In the special case where the curvature effects of the boundary can be neglected, e.g., the tangential derivatives $\nabla_{t}^{2} \tilde{\phi}$ in $\nabla^{2} \tilde{\phi}$ are much smaller than the normal-direction derivatives $\partial_{n}^{2} \tilde{\phi}$, the Poisson-Boltzmann equation (7) can be integrated once. The standard trick is to multiply the equation by $\partial_{n} \tilde{\phi}$, and then to use that $\left(\partial_{n} \tilde{\phi}\right) \partial_{n}^{2} \tilde{\phi}=\partial_{n}\left[\left(\partial_{n} \tilde{\phi}\right)^{2}\right] / 2$ and $-z_{\mathrm{X}}\left(\partial_{n} \tilde{\phi}\right) \exp \left(-z_{\mathrm{X}} \tilde{\phi}\right)=\partial_{n}\left[\exp \left(-z_{\mathrm{X}} \tilde{\phi}\right)\right]$. The result, known as Grahame's equation [55], is

$$
\left(\tilde{\sigma}_{\mathrm{o}}\right)^{2}=\tilde{q}_{I}^{2} \sum_{\mathrm{X}, z_{\mathrm{X}}} \tilde{c}_{\mathrm{X}, z_{\mathrm{X}}}^{\mathrm{b}}\left[e^{-z_{\mathrm{X}} \tilde{\phi}_{\mathrm{d}}}-e^{-z_{\mathrm{X}} \tilde{\phi}_{\mathrm{m}}}\right],
$$

where $\tilde{\phi}_{\mathrm{m}}$ is the potential at the midpoint between opposite walls in the normal direction. In this simplified case, Eqs. (12), (13), and (15) form a self-consistent set of algebraic equations for the determination of $\tilde{\sigma}_{\mathrm{o}}, \tilde{\phi}_{\mathrm{o}}$, and $\tilde{\phi}_{\mathrm{d}}$. This algebraic approach, in particular, can be employed for flat plane-parallel channels of a very large width-to-height aspect ratio, since in this case, the curvature effects only play a role for the vanishingly small region at the edges of the channel cross section.

\section{E. Entrance effects and permselectivity}

For low salt concentration, the electric double layers of opposite walls of the nanochannel begin to overlap. Counterions are attracted to the nanochannel, while coions are expelled, and the nanochannel becomes permselective to the counterions. At each entrance of the nanochannel, the ionic densities have to attain their respective bulk values. This is achieved by displacing ions across the entrance, resulting in an ionic screening layer of width $\lambda_{\mathrm{D}}$ outside the entrance and uncompensated wall charges in the nanochannel, thus, creating oppositely pointing electric dipoles at each entrance and spawning the Donnan potential [2]. For low currents (the linear regime), no additional dissipation is created by this process: The potential drop experienced when passing into the nanochannel through one dipole region is canceled by the potential gain when leaving the nanochannel through the other dipole region.

The linear regime breaks down when a certain critical current $I_{\mathrm{c}}$, the so-called limiting current, is reached. In the limit of low flow velocities, $I_{\mathrm{c}}$ can be estimated by the classic Levich theory $[2,56,57]$ corrected by the logarithmic flux-focusing factor [31],

$$
I_{\mathrm{c}}=\frac{\pi}{\ln \left(\frac{L_{\mathrm{o}}}{2 h}\right)}\left(\frac{\eta+1}{\eta-1}\right) 2 e \mathcal{A}_{\mathrm{res}} D \frac{c_{I}}{L_{\mathrm{o}}},
$$

where $L_{\mathrm{o}}$ is the distance from the nanochannel entrance to the electrode in the reservoir, $2 h$ is the height of the nanochannel, $\eta=I_{+} / I_{-}$is the ratio of counterion and coion currents, $e$ is the elementary charge, $\mathcal{A}_{\text {res }}$ is the reservoir cross-sectional area, $D$ is the ionic diffusivity, and $c_{I}$ is the ionic strength of the solution in the reservoir.

For currents above $I_{\mathrm{c}}$, a finite-sized polarization concentration region with nearly zero ion concentration develops in front of the anode-side entrance of the nanochannel, and there will be a significant voltage drop across this region. When this happens, the measured conductance $G$ of the system drops, and this more strongly for lower reservoir concentration, in stark contrast to the increased $G$ observed on the low concentration side of the conductance minimum given by $\delta$ in Table I and Eq. (21).

In Table III, we list the estimates for the limiting current $I_{\mathrm{c}}$ for the low concentration $\left(c_{\mathrm{KCl}}^{\text {tot }}<0.1 \mathrm{mM}\right)$ data points in Sec. IV B. In our experiments described in Sec. IV, we have

TABLE III. Estimates based on Eq. (16) of the limiting current $I_{\mathrm{c}}$ for data points in Sec. IV B with $c_{\mathrm{KCl}}^{\text {tot }}<0.1 \mathrm{mM}$. $\mathcal{A}_{\text {res }}=1.5 \mathrm{~mm} \times$ $0.5 \mathrm{~mm}, L_{\mathrm{o}}=0.75 \mathrm{~mm}$, and $D=6 \times 10^{-9} \mathrm{~m}^{2} / \mathrm{s}$.

\begin{tabular}{lccccc}
\hline \hline $\begin{array}{c}c_{\mathrm{KCl}}^{\text {tot }} \\
{[\mu \mathrm{M}]}\end{array}$ & $\begin{array}{c}c_{I} \\
{[\mu \mathrm{M}]}\end{array}$ & $\lambda_{\mathrm{D}} / h$ & $-\tilde{\phi}_{\mathrm{m}}$ & $\eta$ & $\begin{array}{c}I_{\mathrm{c}} \\
{[\mathrm{nA}]}\end{array}$ \\
\hline 0.0 & 2.1 & 1.27 & 3.32 & 759 & 1.1 \\
0.1 & 2.2 & 1.24 & 3.28 & 707 & 1.1 \\
0.3 & 2.4 & 1.19 & 3.22 & 619 & 1.2 \\
1.0 & 3.1 & 1.05 & 3.01 & 413 & 1.6 \\
3.0 & 5.1 & 0.82 & 2.61 & 186 & 2.6 \\
10.0 & 12.1 & 0.53 & 1.90 & 44 & 6.3 \\
25.0 & 27.1 & 0.35 & 1.23 & 12 & 16.0 \\
50.0 & 52.1 & 0.26 & 0.73 & 4 & 41.8 \\
\hline \hline
\end{tabular}


made sure that all our measurements were performed in the linear regime by using currents below $0.2 \mathrm{nA}$, i.e., at least a factor of 5 below the estimated limiting current $I_{\mathrm{c}}$. Therefore, the measured conductance is the intrinsic nanochannel conductance. Furthermore, by reversing the polarity, we have checked that no current rectification did take place (data not shown).

\section{F. Nanochannel conductance}

Operating under conditions where the entrance effects are negligible, the electrical conductance $G$ of an aqueous-filled nanochannel is found as $G=I / \Delta V$ by applying a known voltage drop $\Delta V$ and measuring the resulting current $I$. The electromigrative contribution $G_{\mathrm{mig}}=I_{\mathrm{mig}} / \Delta V$ to $G$ is found by integrating over the sum of the conductivities for the chemical families $X$. The external applied voltage also gives rise to an axial electric field $\Delta V / L$ driving an axial electroosmotic flow $v(y, z)$ and resulting in an advective current $I_{\mathrm{adv}}$ with the corresponding contribution, $G_{\mathrm{adv}}=I_{\mathrm{adv}} / \Delta V$, to the conductance. The flow is found by solving the steady-state Navier-Stokes equation with an electrical body force in a rectangular channel [58]. For high-aspect-ratio rectangular channels (height $2 h$ much smaller than width $w$ ), only the vertical $z$ direction matters, and the explicit expression for the total conductance, $G=G_{\mathrm{mig}}+G_{\mathrm{adv}}$, is well approximated by

$$
\begin{aligned}
G= & \frac{2 e w}{L} \sum_{\mathrm{X}, z_{\mathrm{X}}} z_{\mathrm{X}} \mu_{\mathrm{X}, z_{\mathrm{X}}}^{* \mathrm{~b}} c_{\mathrm{X}, z_{\mathrm{X}}}^{\mathrm{b}} \int_{-h}^{0} e^{-z_{\mathrm{X}} \tilde{\phi}(z)} d z \\
& +\frac{2 \epsilon k_{\mathrm{B}} T w}{\eta L} \sum_{\mathrm{X}, z_{\mathrm{X}}} z_{\mathrm{X}} c_{\mathrm{X}, z_{\mathrm{X}}}^{\mathrm{b}} \int_{-h}^{0}\left[\tilde{\phi}(z)-\tilde{\phi}_{\mathrm{d}}\right] e^{-z_{\mathrm{X}} \tilde{\phi}(z)} d z .
\end{aligned}
$$

For zero internal potential $(\tilde{\phi}=0)$, the effects of the wall disappear, and we obtain the bulk conductivity $\sigma_{\mathrm{el}}^{\mathrm{b}}$ as

$$
\sigma_{\mathrm{el}}^{\mathrm{b}}=e \sum_{\mathrm{X}, z_{\mathrm{X}}} z_{\mathrm{X}} \mu_{\mathrm{X}, z_{\mathrm{X}}}^{* \mathrm{~b}} c_{\mathrm{X}, z_{\mathrm{X}}}^{\mathrm{b}} .
$$

\section{NUMERICAL ONE-DIMENSIONAL ANALYSIS}

In the following numerical analysis, we consider the case of a channel aligned with the $x$ axis and with a rectangular cross section in the $y z$ plane of height $2 h$ and width $w$ such that $-h<$ $z<h$ and $0<y<w$, mimicking our experimental system described in Sec. IV. The width-to-height aspect ratio in this case is large, $w \gg 2 h$ so that we can apply a one-dimensional (1D) approximation, where the side walls at $y=0$ and $y=w$ can be neglected, and only the top and bottom walls at $z= \pm h$ play a role.

\section{A. The numerical algorithm}

The first part of our numerical scheme is using the buffer calculator [49] based on Eq. (2) to determine the bulk concentrations $c_{\mathrm{X}, z_{\mathrm{X}}}^{\mathrm{b}}$ for a given solution of the ions $\mathrm{H}^{+}$, $\mathrm{OH}^{-}, \mathrm{HCO}_{3}^{-}, \mathrm{CO}_{3}^{2-}, \mathrm{K}^{+}$, and $\mathrm{Cl}^{-}$. We then consider the self-consistent solution of Eqs. (12), (13), and (15), which, in this case, takes the form

$$
\tilde{\sigma}_{\mathrm{o}}=\tilde{C}_{\mathrm{s}}\left(\tilde{\phi}_{\mathrm{o}}-\tilde{\phi}_{\mathrm{d}}\right)
$$

$$
\begin{aligned}
\tilde{\sigma}_{\mathrm{o}} & =\frac{e^{-2 \tilde{\phi}_{\mathrm{o}}}-\tilde{K}_{-} \tilde{K}_{+}}{\tilde{K}_{-} \tilde{K}_{+}+\tilde{K}_{+} e^{-\tilde{\phi}_{\mathrm{o}}}+e^{-2 \tilde{\phi}_{\mathrm{o}}}}, \\
\left(\tilde{\sigma}_{\mathrm{o}}\right)^{2} & =\tilde{q}_{I}^{2} \sum_{\mathrm{X}, z_{\mathrm{X}}} \tilde{c}_{\mathrm{X}, z_{\mathrm{X}}}^{\mathrm{b}}\left[e^{-z_{\mathrm{X}} \tilde{\phi}_{\mathrm{d}}}-e^{-z_{\mathrm{X}} \tilde{\phi}_{\mathrm{m}}}\right],
\end{aligned}
$$

together with the 1D Poisson-Boltzmann equation for planar walls including boundary conditions at the wall, $z=-h$, and at the center of the channel, $z=0$,

$$
\begin{gathered}
\partial_{z}^{2} \tilde{\phi}=-\frac{1}{2 \lambda_{\mathrm{D}}^{2}} \sum_{\mathrm{X}, z_{\mathrm{X}}} z_{\mathrm{X}} \tilde{c}_{\mathrm{X}, z_{\mathrm{X}}}^{\mathrm{b}} e^{-z_{\mathrm{X}} \tilde{\phi}}, \\
\tilde{\phi}(-h)=\tilde{\phi}_{\mathrm{d}}, \quad \partial_{z} \tilde{\phi}(0)=0 .
\end{gathered}
$$

We implement and solve the problem using MATLAB (Mathworks, Inc.) using the following self-consistent iteration algorithm:

Initialization: Calculate $\mathrm{pH}=-\log _{10}\left(c_{\mathrm{H}}^{\mathrm{b}}\right)$ and ionic concentrations $\tilde{c}_{\mathrm{X}, z_{\mathrm{X}}}^{\mathrm{b}}$ in the bulk using the buffer calculator [49] Eq. (2), and assume $\tilde{\phi}_{\mathrm{m}}=0$.

Loop: Solve Eq. (19) for $\tilde{\sigma}_{\mathrm{o}}, \tilde{\phi}_{\mathrm{o}}$, and $\tilde{\phi}_{\mathrm{d}}$, then solve Eq. (20) for $\tilde{\phi}$, and finally obtain $\tilde{\phi}_{\mathrm{m}}=\tilde{\phi}(0)$.

Test: Repeat the loop until $\tilde{\phi}_{\mathrm{m}}$ has converged.

Once converged, the algorithm provides the potential $\tilde{\phi}(z)$ to be used in Eq. (17) for calculating the conductance $G$. This algorithm, which self-consistently couples the $\mathrm{CO}_{2}$-induced hydronium, surface, and bulk reactions, as well as nonlinear electrokinetics of the double layer, is the first main theoretical result of this paper.

\section{B. Prediction of the conductance minimum}

The second theoretical result is the model prediction that the conductance $G$ of a nanochannel depends nonmonotonically on the $\mathrm{KCl}$ concentration $c_{\mathrm{KCl}}^{\text {tot }}=c_{\mathrm{K}^{+}}^{\mathrm{b}}=c_{\mathrm{Cl}^{-}}^{\mathrm{b}}$ in the reservoirs. The nanochannel parameters listed in Table IV are chosen to correspond to our experimental validation presented in Sec. IV, and the simulation results are displayed in Fig. 2. Consistent with literature, the modeled conductance of the nanochannel follows a linear relation as does measured bulk conductance at high salt concentrations. Furthermore, the modeled conductance reaches a plateau in the infinite-dilution limit, similar to that found by Refs. [19,24,28,45]. However, as indicated in the log-log plot of Fig. 2(a) by the labels Plateau, Valley, Departure, and Bulk, we find, in contrast to these previous model studies, but in line with Ref. [32] where it went

TABLE IV. List of the nanochannel parameters used in the model calculation of the nanochannel conductance, Fig. 2.

\begin{tabular}{lcc}
\hline \hline Parameter & Symbol & Value \\
\hline Height & $2 h$ & $165 \mathrm{~nm}$ \\
Width & $w$ & $8.3 \mu \mathrm{m}$ \\
Length & $L$ & $12.0 \mathrm{~mm}$ \\
Surface site density & $\Gamma_{\text {tot }}$ & $5 \mathrm{~nm}^{-2}$ \\
Stern capacitance & $C_{\mathrm{s}}$ & $0.2 \mathrm{~F} \mathrm{~m}^{-2}$ \\
Viscosity of water & $\eta$ & $1.0 \mathrm{mPa} \mathrm{s}^{-1}$ \\
Permittivity of water & $\epsilon$ & $690 \mathrm{pF} \mathrm{m}^{-1}$ \\
Temperature & $T$ & $298 \mathrm{~K}$ \\
\hline \hline
\end{tabular}



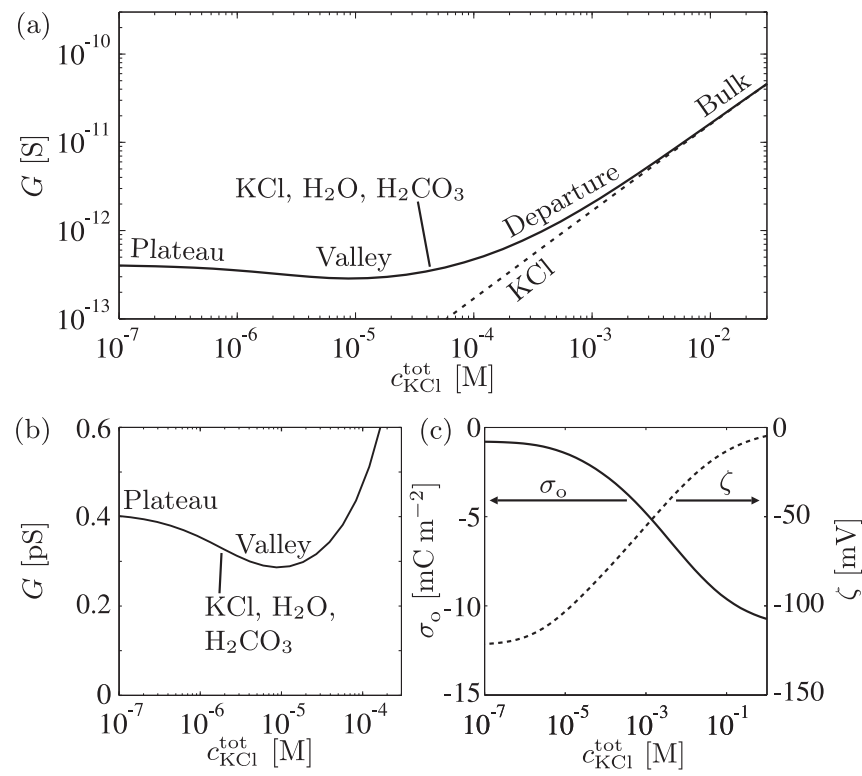

FIG. 2. (Color online) Theoretical predictions for the nanochannel conductance $G$ Eq. (17) vs $\mathrm{KCl}$ concentration $c_{\mathrm{KCl}}^{\text {tot }}$ in the reservoirs for the system described in Table IV and Sec. IV. (a) Log-log plot of $G$ vs $c_{\mathrm{KCl}}^{\text {tot }}$ taking $\mathrm{KCl}$, dissolved $\mathrm{CO}_{2}$, and hydronium ions (full line) into account, and bulk conductance taking only $\mathrm{KCl}$ into account (dashed line). Four conductance regimes are marked: bulk, departure, valley (minimum conductance), and plateau. (b) The nonmonotonic conductance from panel (a) in a lin-log plot. (c) The $c_{\mathrm{KCl}}^{\text {tot }}$ dependence of the $\zeta$ potential $\zeta=\phi_{\mathrm{d}}$ and the surface charge $\sigma_{\mathrm{o}}$.

un-noticed, that our model predicts a nonmonotonic behavior: A minimum conductance (the valley) is obtained before reaching the plateau. This conductance minimum positioned at $c_{\mathrm{KCl}}^{\mathrm{tot}}=10 \mu \mathrm{M}$ is more clearly pronounced in the lin-log plot of Fig. 2(b). We quantify the magnitude of the conductance minimum as the ratio $\delta$ of the largest conductance $G_{\max }$ on the low concentration side of the minimum and the minimum conductance $G_{\min }$,

$$
\delta=\frac{G_{\max }}{G_{\min }} .
$$

For the KCl-based Fig. 2(b), we calculate $\delta_{\mathrm{KCl}}=1.42$.

In Fig. 2(c), we show how the calculated $\zeta$ potential $\phi_{\mathrm{d}}$ and surface charge $\sigma_{\mathrm{o}}$ vary with salt concentration, trends that have been previously observed theoretically and have been validated experimentally [26,44]. Moreover, as the magnitude of the $\zeta$ potential is small for our model system, $|\zeta|<120 \mathrm{mV} \approx$ $5 k_{\mathrm{B}} T / e$, we have justified our neglecting of ionic crowding effects near the surface [59], and we gain confidence in our model results.

\section{Characterization of the conductance minimum}

Further results of our model showing nonmonotonic conductance vs the reservoir salt concentration $c_{\mathrm{KCl}}^{\text {tot }}$ are presented in Fig. 3, where parameters of the problem are varied one by one, relative to those of Fig. 2, which here and in the following are denoted $G_{\mathrm{o}}$ (full black curve). In particular, when $\mathrm{CO}_{2}$ is not included in our model (dashed red curve), the conductance changes by up to a factor of 2 relative to $G_{\mathrm{o}}$. Additionally, without $\mathrm{CO}_{2}$, the position of the conductance minimum shifts

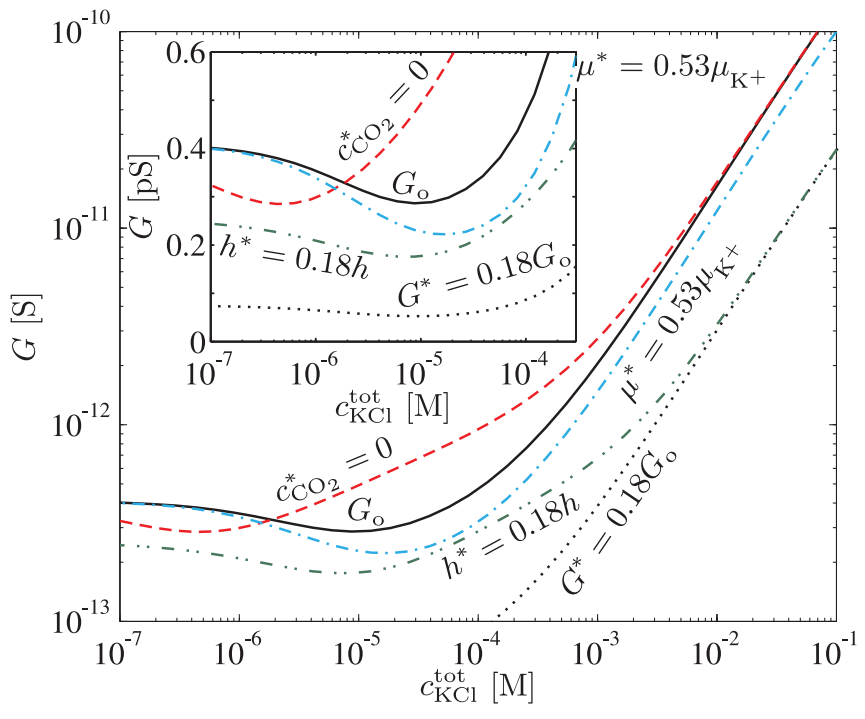

FIG. 3. (Color online) Theoretical prediction for the nanochannel conductance $G$ Eq. (17) vs $c_{\mathrm{KCl}}^{\text {tot }}$ in the reservoirs for the parameters of Fig. $2\left(G_{0}\right.$, full black curve) and for variations from that: removal of $\mathrm{CO}_{2}\left(c_{\mathrm{CO}_{2}}^{*}=0\right.$, dashed red curve), decreased mobility of the counterions $\left(\mu^{*}=0.53 \mu_{\mathrm{K}^{+}}=\mu_{\mathrm{Li}^{+}}\right.$, dot-dashed blue curve), and decreased nanochannel height $\left(h^{*}=0.18 h\right.$, dot-dot-dashed green curve). Also shown is a scaled curve $\left(G^{*}=0.18 G_{\mathrm{o}}\right.$, dotted black curve) for aid in comparison to the reduction of nanochannel height. The inset shows the nonmonotonic conductance in a lin-log plot, which enhances the predicted features.

from $c_{\mathrm{KCl}}^{\text {tot }} \approx 10 \mu \mathrm{M}$ down to $\approx 0.4 \mu \mathrm{M}$ governed solely by autoprotolysis of water, again showing the importance of $\mathrm{CO}_{2}$ induced hydronium ions for the appearance of nonmonotonic conductance.

In Fig. 3, we also plot $G$ of a solution with a lower ion mobility. Specifically, we substitute $\mathrm{KCl}$ with $\mathrm{LiCl}$ (dotdashed blue curve), thereby reducing the counterion mobility by a factor of 0.53 from $\mu_{\mathrm{K}^{+}}=76.2 \times 10^{-9} \mathrm{~m}^{2}(\mathrm{~V} \mathrm{~s})^{-1}$ to $\mu^{*}=\mu_{\mathrm{Li}^{+}}=40.1 \times 10^{-9} \mathrm{~m}^{2}(\mathrm{~V} \mathrm{~s})^{-1}$. Mobility reduction implies that: (i) In the high $c_{\mathrm{KCl}}^{\text {tot }}$ regime $\sim 10^{-3}-10^{-1} \mathrm{M}$, the conductance is lower by a factor of $\sim 0.75$ consistent with the lower bulk conductivity of the $\mathrm{LiCl}$ electrolyte. (ii) In the very dilute $c_{\mathrm{KCl}}^{\text {tot }}$ regime $\sim 10^{-7}-10^{-6} \mathrm{M}$, the conductance plateau reaches the same conductance value as $\mathrm{KCl}$, again verifying that nanochannel conductance in this regime is dominated by the contribution from $\mathrm{CO}_{2}$-induced hydronium. (iii) In the intermediate $c_{\mathrm{KCl}}^{\text {tot }}$ regime $\sim 10^{-6}-10^{-3} \mathrm{M}$, the conductance curve has a different shape: The position of the minimum is shifted to a higher value of salt concentration, $c_{\mathrm{KCl}}^{\text {tot }}=20 \mu \mathrm{M}$, and its magnitude is increased from $\delta_{\mathrm{KCl}}=1.42$ to $\delta_{\mathrm{LiCl}}=$ 1.77. This enhancement of the conductance minimum for the lower-mobility counterion $\mathrm{Li}^{+}$is consistent with the intake of counterions and the expulsion of coions in nanochannels with overlapping electric double layers, a situation where the counterions dominate the conductance.

We also show the dependence of nanochannel height on the predominance of a nonmonotonic conductance at low concentrations in Fig. 3. Specifically, we reduce the height of the nanochannel by a factor of 0.18 to $2 h^{*}=30 \mathrm{~nm}$ (dot-dot-dashed green curve), corresponding to the silica 
nanochannels of Ref. [60]. Again, we note that: (i) In the high $c_{\mathrm{KCl}}^{\text {tot }}$ regime $\sim 10^{-3}-10^{-1} \mathrm{M}$, the conductance is lower by a factor of $\sim 0.18$ consistent with the reduced cross-sectional area. (ii) In the intermediate $c_{\mathrm{KCl}}^{\text {tot }}$ regime $\sim 10^{-5}-10^{-3} \mathrm{M}$, the conductance curve has a more linear slope and, therefore, a wide regime of minimum conductance. (iii) In the very dilute $c_{\mathrm{KCl}}^{\mathrm{tot}}$ regime $\sim 10^{-7}-10^{-5} \mathrm{M}$, the conductance is lower only by a factor of $\sim 0.6$ and not by the aforementioned factor of 0.18 . This relatively higher conductance in smaller channels at low concentrations is due to overlapping electric double layers. For a binary symmetric electrolyte, the conductivity in the electric double layer $\sigma_{\mathrm{el}}^{\mathrm{DL}}$ will be higher than the bulk conductivity if the $\zeta$ potential is larger than the thermal voltage $\zeta>k_{\mathrm{B}} T /(z e)$ simply due to an increased ionic concentration. This is indeed the case in our nanochannels, especially at low concentrations, as seen in Fig. 2(c). Hence, in our nanochannels, as the concentration is lowered, the conductivity in the channel gradually changes from $\sigma_{\mathrm{el}}^{\mathrm{b}}$ to $\sigma_{\mathrm{el}}^{\mathrm{DL}}$ concurrently with the electric double layer overlap. The smaller the channel dimensions, the greater the overlap, and, thus, the higher the average conductivity at a given low concentration. Note that, for aid in comparing this low-height curve with the nominal conductance curve, we also have plotted the scaled version $G^{*}=0.18 G_{\mathrm{o}}$ (dotted black curve) of the original curve.

These theoretical model predictions must now be verified experimentally. To date, log-log plots of conductance vs salt concentration in unbuffered solutions do not readily show the conductance valley, but much of this may be because such a valley is difficult to discern in a log-log plot, as shown in Figs. 2 and 3. However, as listed in Table I, we have found six examples in the literature of an observed (but un-noticed) conductance minimum. To provide further experimental validation of our theoretical predictions, we performed a set of conductance measurements on silica nanochannels described in Sec. IV, varying just one parameter, namely, the hydronium concentration through the addition of $\mathrm{HCl}$, to show that not only does this valley exist, but also our model predicts the behavior of the valley accurately when other ions are added to the system.

\section{EXPERIMENTAL VALIDATION}

To test our theoretical predictions, we performed experiments in both bulk solutions and fused-silica nanochannels fabricated in-house at the University of California, Santa Barbara [26]. The nanochannels (165-nm high, 8.3- $\mu \mathrm{m}$ wide, and 12-mm long) were equipped with 1.5-mm-diameter reservoirs of depth $0.5 \mathrm{~mm}$ and mounted as shown in Fig. 4(a). We prepared $0.2-\mu \mathrm{m}$-filtered $\mathrm{KCl}$ concentrations $c_{\mathrm{KCl}}^{\text {tot }}=0.0001$, $0.0003,0.001,0.003,0.01,0.025,0.05,0.1,0.5,1,5,10$, and $100 \mathrm{mM}$ and another set with $50 \mu \mathrm{M}$ hydrochloric acid added at the same $\mathrm{KCl}$ concentrations and at the additional values $c_{\mathrm{KCl}}^{\text {tot }}=0.02,0.2,0.6$, and $50 \mathrm{mM}$.

\section{A. Measured bulk conductance}

To validate the bulk conductance part of our model, we conducted bulk measurements using a standard technique: The prepared solutions were pipetted into the sample chamber of

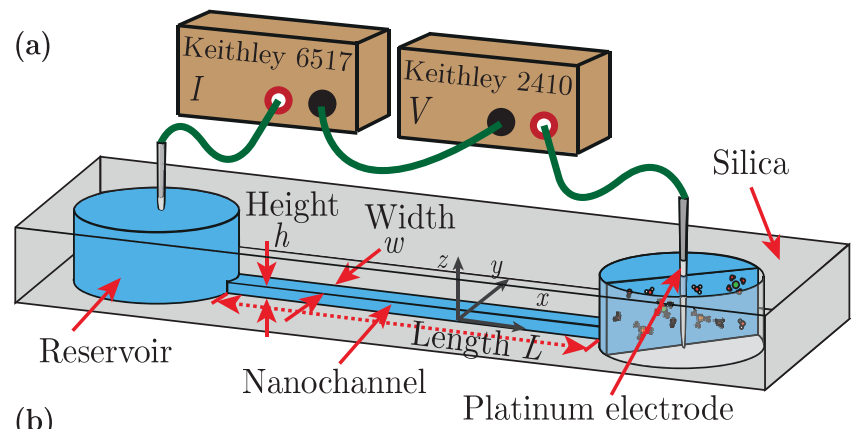

(b)

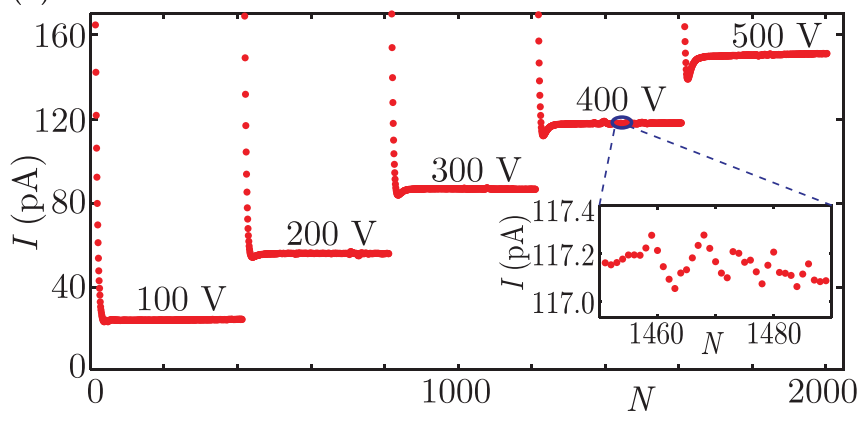

FIG. 4. (Color online) Experimental measurement of current $I$ in silica nanochannels of dimensions $h \times w \times L=165 \mathrm{~nm} \times$ $8.3 \mu \mathrm{m} \times 12 \mathrm{~mm}$. (a) Our setup. For each experiment, the voltage $\Delta V$ (from a Keithley 2410) was applied for 5-10 min to allow $I$ to equilibrate (monitored by a high-sensitive electrometer, Keithley 6517). (b) Raw data (dots) of $I$ vs sample number $N$ for the five values of $\Delta V$ indicated. The inset shows detailed variation of $I$ vs $N$ at 8 samples/s.

a commercial $\mathrm{pH}$ and conductivity meter (Oakton, Inc), and readouts of the $\mathrm{pH}$ and conductivity values were performed after allowing for equilibration to have taken place. These measurements were performed periodically on the prepared solutions to make sure that variations due to temperature and humidity did not exceed $5 \%$ of the original value. All solutions were filtered with $0.2-\mu \mathrm{m}$ PTFE syringe filters prior to use.

Figure 5(a) shows the measured bulk conductivity for the $\mathrm{KCl}$ solutions used in our study, together with two model calculations of $\sigma_{\mathrm{el}}^{\mathrm{b}}$ based on Eq. (18) and Table II without any additional adjustable parameters: one (full line) taking into account all ions $\left(\mathrm{H}^{+}, \mathrm{OH}^{-}, \mathrm{HCO}_{3}^{-}, \mathrm{CO}_{3}^{2-}, \mathrm{K}^{+}, \mathrm{Cl}^{-}\right)$, the other (dashed line) only including the salt $\left(\mathrm{K}^{+}, \mathrm{Cl}^{-}\right)$. Both measurements and model calculations show the expected monotonic decrease as a function of the bulk $\mathrm{KCl}$ concentration $c_{\mathrm{KCl}}^{\text {tot }}$ with a crossover to a constant value at $c_{\mathrm{KCl}}^{\text {tot }} \approx 5 \times 10^{-6} \mathrm{M}$. The inset shows a scatter of the measurements relative to the model with errors within $13 \%$, which, thus, constitutes an estimate of the experimental uncertainty in our paper. To further test the model, we added $50-\mu \mathrm{M} \mathrm{HCl}$ to our $\mathrm{KCl}$ solutions; experimental results and model calculation are shown in Fig. 5(b), with the inset indicating the deviation of measurements relative to the model to be within $16 \%$.

\section{B. Measured nanochannel conductance}

The nanochannel conductance was measured as follows. After electrokinetically rinsing each channel thoroughly with DI water three times, we applied five voltages to the system 


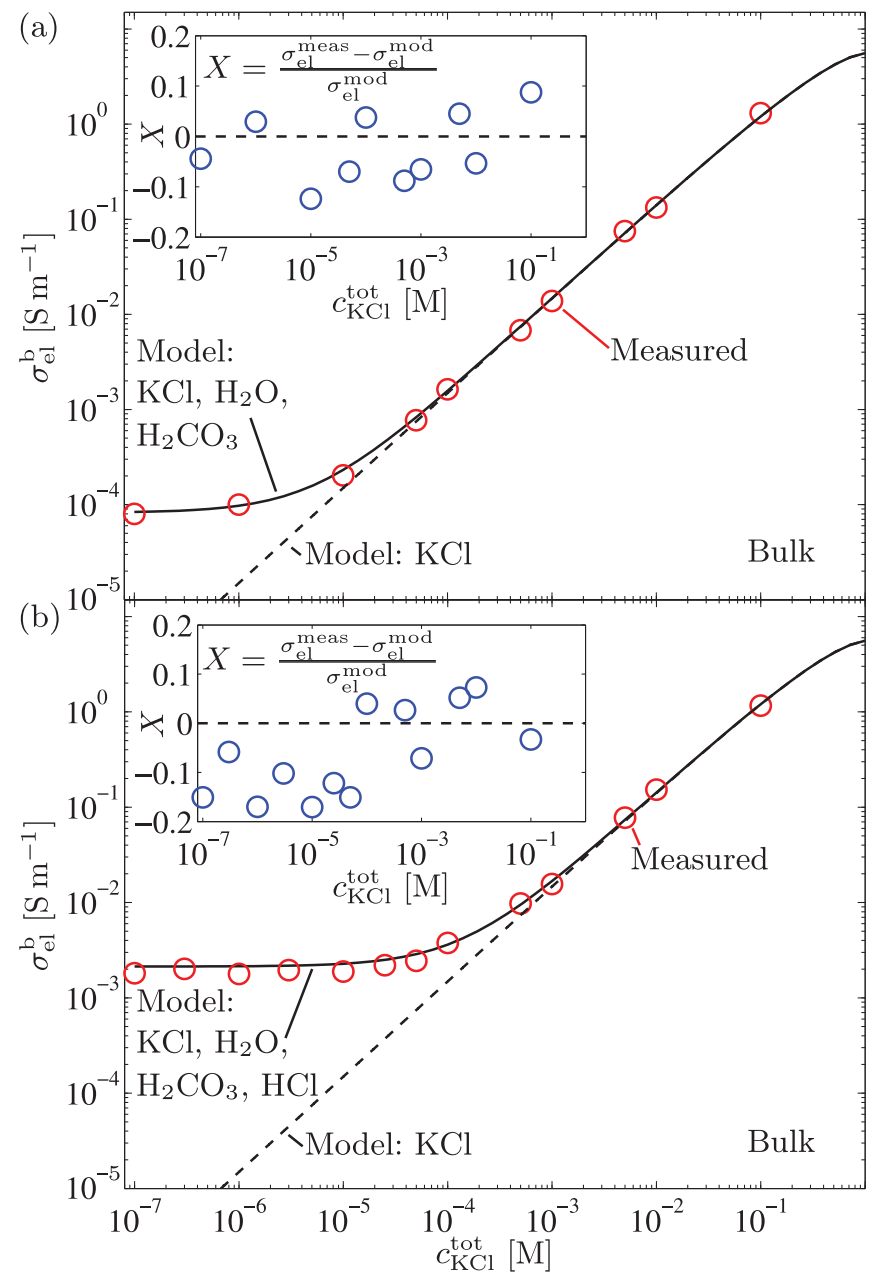

FIG. 5. (Color online) (a) Conductivity $\sigma_{\text {el }}^{\mathrm{b}}$ vs $\mathrm{KCl}$ concentration $c_{\mathrm{KCl}}^{\text {tot }}$ for bulk measurements ( $\sigma_{\mathrm{el}}^{\text {meas }}$, open red circles) and model calculations for both the full model with $\mathrm{KCl}, \mathrm{H}_{2} \mathrm{CO}_{3}$, and $\mathrm{H}_{2} \mathrm{O}\left(\sigma_{\mathrm{el}}^{\mathrm{mod}}\right.$, full line) and for a partial model with only $\mathrm{KCl}$ (dashed line). Model calculations are based on Eq. (18) and Table II without any additional adjustable parameters. The inset shows the relative deviation $X=$ $\left(\sigma_{\mathrm{el}}^{\text {meas }}-\sigma_{\mathrm{el}}^{\text {mod }}\right) / \sigma_{\mathrm{el}}^{\text {mod }}$ (open blue circles) of the measurements from the model. (b) Same as panel (a) with the addition of 50- $\mu \mathrm{M} \mathrm{HCl}$.

$(100,200,300,400$, and $500 \mathrm{~V})$ in succession, each one lasting approximately $40 \mathrm{~s}$. Raw data are shown in Fig. 4(b). To ensure consistent and repeatable data, if the trial did not yield four or five results that were within $10 \%$ of each other, we assumed an unsteady current reading, corroded electrodes, external interference, or poor channel preparation, and discarded the data. Solution exchanges were performed in a systematic fashion to avoid introducing bubbles or particulate matter into the channels.

Moreover, for concentrations below $10 \mu \mathrm{M}$, we covered the system with a Faraday cage to limit measurement noise. After each day of experiments, we flushed the reservoirs multiple times with DI water to prevent a buildup of salt deposits on the channel during its time in storage, and then stored the chip in a dry Eppendorf tube housed in a nitrogen container. We note that electrolysis does not affect the current measurements in our system: Conservatively, we estimate the $\mathrm{pH}$ change of the reservoir to be $7.7 \times 10^{-4} \mathrm{~s}^{-1}$ [48]; next, we find the

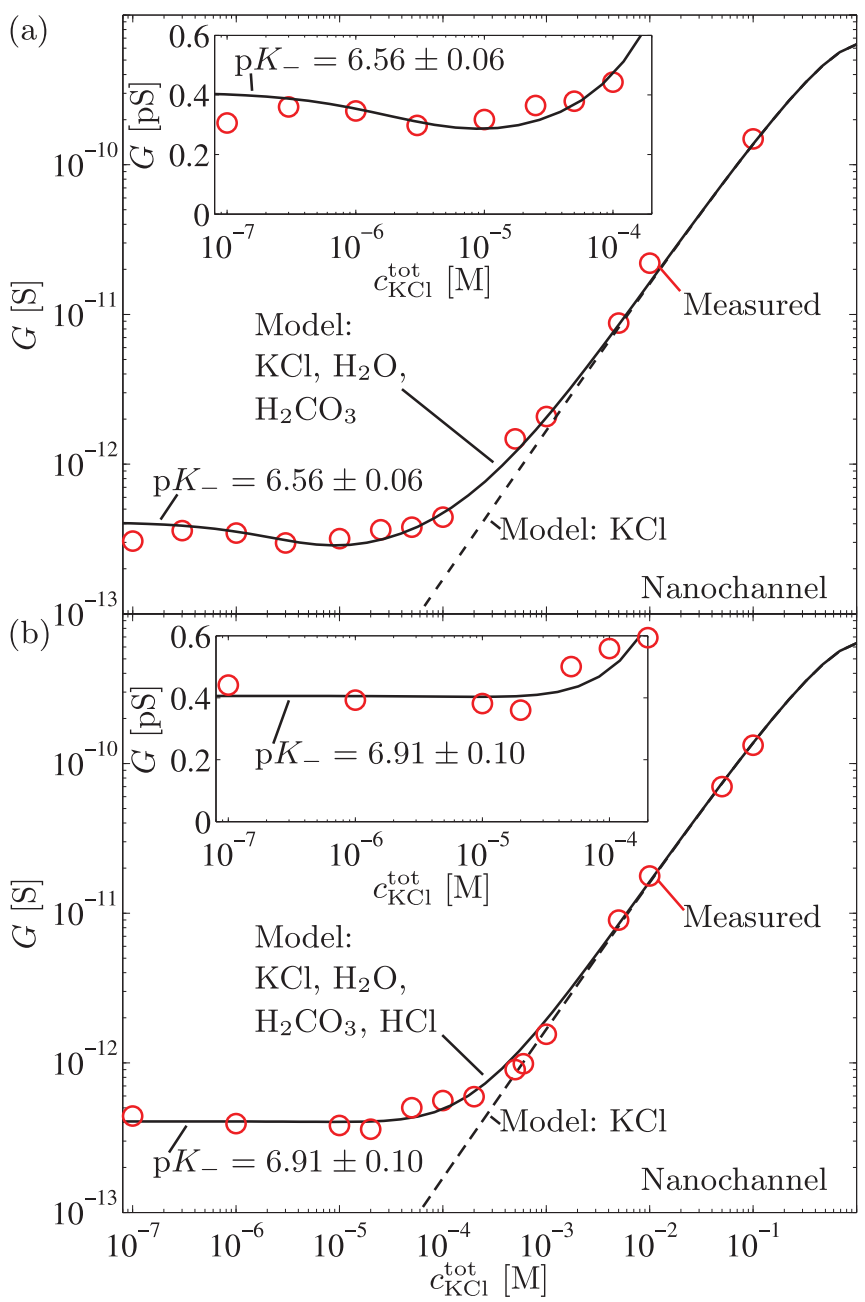

FIG. 6. (Color online) Log-log plot of measured nanochannel conductance $G$ vs $\mathrm{KCl}$ concentration $c_{\mathrm{KCl}}^{\text {tot }}$ (red circles) and curve fits based on Eq. (17) using the logarithmic variables $\log _{10}(G)$ and $\log _{10}\left(c_{\mathrm{KCl}}^{\mathrm{tot}}\right)$ for model electrolytes of various compositions (full and dashed curves) employing the parameters of Tables II and IV. (a) No $\mathrm{HCl}$ added: Fitting to a model including $\mathrm{KCl}, \mathrm{H}_{2} \mathrm{O}$, and $\mathrm{H}_{2} \mathrm{CO}_{3}$ (full curve) yields $\mathrm{p} K_{-}=6.56 \pm 0.06$. Also shown is a model calculation including only $\mathrm{KCl}$ (dashed line). (b) As before but with $50 \mu \mathrm{M}$ of $\mathrm{HCl}$ added: Fitting to a model including $\mathrm{KCl}, \mathrm{HCl}, \mathrm{H}_{2} \mathrm{O}$, and $\mathrm{H}_{2} \mathrm{CO}_{3}$ (full curve) yields $\mathrm{p} K_{-}=6.91 \pm 0.10$. The insets are lin-log plots of the respective low salt concentration regions.

volumetric flow rate to be $4 \times 10^{-15} \mathrm{~m}^{3} / \mathrm{s}$; furthermore, the absorption of $\mathrm{CO}_{2}$ in the reservoir gives rise to $\mathrm{H}_{2} \mathrm{CO}_{3}$, which acts as a buffer and would further prevent $\mathrm{pH}$ changes, and finally, we replenished the reservoirs every $3 \mathrm{~min}$.

Due to a significant electro-osmotic flow velocity in our 165-nm-high nanochannels, we do not encounter the hour-long equilibration time for conductance measurements encountered in 2-nm-high nanochannels [32].

The most significant contribution of our model is predicting the nanochannel conductance, which is shown together with experimental measurements in Fig. 6. Panel (a) shows results for $\mathrm{KCl}$ solutions between infinite dilution (DI water) and $100 \mathrm{mM}$. Using $\mathrm{p} K_{-}$as the only fitting parameter, and using $\log _{10}\left(c_{\mathrm{KCl}}^{\text {tot }}\right)$ and $\log _{10}(G)$ as variables to ensure an even weighting between low and high concentration values, we 
obtain the fit shown for $\mathrm{p} K_{-}=6.56 \pm 0.06$, corresponding well to the literature value of 6.64 [44]. The inset expands on the low salt concentration region and shows the measured conductance minimum and the theoretical fitting curve. The lowest concentration point is furthest off this curve, but it is also the one most prone to error. Previous models assuming constant surface charge give the plateau but no valley $[19,24$, $28,45]$, while relaxing this assumption taking surface chemical reactions into account leads to a monotonically decreasing conductance [61]. Notably, the experimental data of previous studies listed in Table I, have shown the same conductance behavior as our model and data, supporting our claim that the inclusion of hydronium ions in our model is consistent with a broad range of experimental results. In all of these references, the experimentally measured conductance has a minimum that the applied models do not capture. Furthermore, the minimum is located at $\mathrm{KCl}$ concentrations around $10^{-5}-10^{-4} \mathrm{M}$ that is consistent with our model predictions.

Another way to validate the predictions of our model is to add an extra amount of hydronium ions. The conductance valley is due to the concentration of $\mathrm{K}^{+}$and $\mathrm{Cl}^{-}$dropping below that of $\mathrm{H}^{+}$and $\mathrm{HCO}_{3}{ }^{-}$together with the fact that the Debye length and $\zeta$ potential increase for decreasing ionic strength (Fig. 2). Therefore, as in Fig. 5, we can test the model by adding $\mathrm{HCl}$ and can observe the change in the conductance curve. The result in Fig. 6(b) shows no valley in both experiment and model. The fit now gives $\mathrm{p} K_{-}=6.91$, indicating that surface reactions are $\mathrm{pH}$ dependent.

Finally, as a control experiment, we measured the change in $\mathrm{pH}$ and conductance of nanochannels substituting $\mathrm{CO}_{2}$ saturated by $\mathrm{CO}_{2}$-oversaturated $\mathrm{KCl}$ solutions generated by dry ice vapors. In this case, $\mathrm{pH}$ dropped by $22 \%$, and $G$ increased by $27 \%$ (data not shown). Clearly, it would be desirable to design experiments with a more controlled content of $\mathrm{CO}_{2}$ in the atmosphere to further investigate its influence on nanochannel conductance, but this is beyond the scope of this current paper.

\section{CONCLUSION}

In this paper, we have extended a recent self-consistent theoretical model of nanochannel ion transport [26] to unbuffered solutions with low salt concentration by taking hydronium ions into account, induced by carbonic acid arising from dissolved $\mathrm{CO}_{2}$ from the atmosphere, through the explicit addition of $\mathrm{H}^{+}$and $\mathrm{HCO}_{3}^{-}$in our coupled solution/wall chemical-equilibrium dissociation scheme. Using this model, we have predicted the hydronium dependence of the electrical conductance of electrolyte-filled nanochannels, and, in particular, we have identified a conductance minimum in the low salt concentration regime as a function of the reservoir salt concentration $c_{\mathrm{KCl}}^{\text {tot }}$. We studied the dependence of the conductance minimum theoretically and have predicted that its magnitude would be reduced dramatically in a $\mathrm{CO}_{2}$-poor atmosphere and significantly enhanced using low-mobility counterions.

Our theoretical prediction of the conductance minimum is supported by several independent experimental observations in the literature. Furthermore, to validate our theoretical predictions, we have successfully compared our model with direct experimental conductance measurements on a wide range of $\mathrm{KCl}$ concentrations with and without the addition of a specific extra amount of hydronium ions from $\mathrm{HCl}$ in $165-\mathrm{nm}-$ high silica nanochannels. A more thorough experimental study is underway to fully characterize the nanochannel conductance minimum at low salt concentrations and $\mathrm{CO}_{2}$-dissolution controlled hydronium concentrations.

Our modeling and experimental results indicate the possibility for developing a sensitive nanochannel-based carbon dioxide sensor. To this end, an in-depth experimental study must be performed in a tightly regulated atmosphere. Combining such measurements with our model could be used to determine which parameter values lead to the most pronounced conductance minimum and what consequences this may have for using nanochannels for unique gas sensors.
[1] T. M. Squires and S. R. Quake, Rev. Mod. Phys. 77, 977 (2005).

[2] R. Schoch, J. Han, and P. Renaud, Rev. Mod. Phys. 80, 839 (2008).

[3] W. Sparreboom, A. van den Berg, and J. C. T. Eijkel, Nat. Nanotechnol. 4, 713 (2009).

[4] L. Bocquet and E. Charlaix, Chem. Soc. Rev. 39, 1073 (2010).

[5] M. Napoli, J. C. T. Eijkel, and S. Pennathur, Lab Chip 10, 957 (2010).

[6] J. Han and H. G. Craighead, Science 288, 1026 (2000).

[7] L. R. Huang, J. O. Tegenfeldt, J. J. Kraeft, J. C. Sturm, R. H. Austin, and E. C. Cox, Nat. Biotechnol. 20, 1048 (2002).

[8] R. Karnik, K. Castelino, R. Fan, P. Yang, and A. Majumdar, Nano Lett. 5, 1638 (2005).

[9] R. Karnik, K. Castelino, and A. Majumdar, Appl. Phys. Lett. 88, 123114 (2006).

[10] J. Fu, R. B. Schoch, A. L. Stevens, S. R. Tannenbaum, and J. Han, Nat. Nanotechnol. 2, 121 (2007).
[11] N. F. Y. Durand and P. Renaud, Lab Chip 9, 319 (2009).

[12] W. Reisner, N. B. Larsen, A. Silahtaroglu, A. Kristensen, N. Tommerup, J. O. Tegenfeldt, and H. Flyvbjerg, Proc. Natl. Acad. Sci. USA 107, 13294 (2010).

[13] H. Daiguji, P. Yang, A. J. Szeri, and A. Majumdar, Nano Lett. 4, 2315 (2004).

[14] W. Olthuis, B. Schippers, J. Eijkel, and A. van den Berg, Sens. Actuators B 111-112, 385 (2005).

[15] F. H. J. van der Heyden, D. J. Bonthuis, D. Stein, C. Meyer, and C. Dekker, Nano Lett. 7, 1022 (2007).

[16] S. Pennathur, J. C. T. Eijkel, and A. van den Berg, Lab Chip 7, 1234 (2007)

[17] C. Davidson and X. Xuan, Electrophoresis 29, 1125 (2008).

[18] M. Wang and Q. Kang, Microfluid. Nanofluid. 9, 181 (2009).

[19] D. Stein, M. Kruithof, and C. Dekker, Phys. Rev. Lett. 93, 035901 (2004).

[20] S. Pennathur and J. G. Santiago, Anal. Chem. 77, 6782 (2005). 
[21] H. Daiguji, P. Yang, and A. Majumdar, Nano Lett. 4, 137 (2004).

[22] K. G. H. Janssen, H. T. Hoang, J. Floris, J. de Vries, N. R. Tas, J. C. T. Eijkel, and T. Hankemeier, Anal. Chem. 80, 8095 (2008).

[23] M. Whitby, L. Cagnon, M. Thanou, and N. Quirke, Nano Lett. 8, 2632 (2008).

[24] D. Martins, V. Chu, D. M. F. Prazeres, and J. P. Conde, Procedia Chemistry (Weinheim, Germany) 1, 1095 (2009).

[25] M. Wang, Q. Kang, and E. Ben-Naim, Anal. Chim. Acta 664, 158 (2010).

[26] M. B. Andersen, J. Frey, S. Pennathur, and H. Bruus, J. Colloid Interface Sci. 353, 301 (2011).

[27] Q. Pu, J. Yun, H. Temkin, and S. Liu, Nano Lett. 4, 1099 (2004).

[28] R. Karnik, R. Fan, M. Yue, D. Li, P. Yang, and A. Majumdar, Nano Lett. 5, 943 (2005).

[29] R. Karnik, C. Duan, K. Castelino, H. Daiguji, and A. Majumdar, Nano Lett. 7, 547 (2007).

[30] S. J. Kim, Y.-C. Wang, J. H. Lee, H. Jang, and J. Han, Phys. Rev. Lett. 99, 044501 (2007).

[31] G. Yossifon, P. Mushenheim, Y.-C. Chang, and H.-C. Chang, Phys. Rev. E 79, 046305 (2009).

[32] C. Duan and A. Majumdar, Nat. Nanotechnol. 5, 848 (2010).

[33] C. G. Armistead, A. J. Tyler, F. H. Hambleton, S. A. Mitchell, and J. A. Hockey, J. Phys. Chem. 73, 3947 (1969).

[34] S. Levine and A. L. Smith, Discuss. Faraday Soc. 52, 290 (1971).

[35] D. E. Yates, S. Levine, and T. W. Healy, J. Chem. Soc., Faraday Trans. 1 70, 1807 (1974).

[36] J. A. Davis, R. O. James, and J. O. Leckie, J. Colloid Interface Sci. 63, 480 (1978).

[37] J. A. Davis and J. O. Leckie, J. Colloid Interface Sci. 67, 90 (1978).

[38] T. Hiemstra, W. V. Riemsdijk, and G. Bolt, J. Colloid Interface Sci. 133, 91 (1989).

[39] R. Charmas, W. Piasecki, and W. Rudzinski, Langmuir 11, 3199 (1995).

[40] N. L. Burns, J. Colloid Interface Sci. 183, 249 (1996).

[41] J. Lützenkirchen, Environ. Sci. Technol. 32, 3149 (1998).
[42] S. H. Behrens and D. G. Grier, J. Chem. Phys. 115, 6716 (2001).

[43] P. M. Biesheuvel, J. Colloid Interface Sci. 275, 514 (2004).

[44] M. Wang and A. Revil, J. Colloid Interface Sci. 343, 381 (2010).

[45] R. B. Schoch and P. Renaud, Appl. Phys. Lett. 86, 25311 (2005).

[46] L. Cheng, Ph.D. thesis, University of Michigan, 2008.

[47] R. F. Probstein, Physicochemical Hydrodynamics: An Introduction (Wiley Interscience, 1994).

[48] A. Persat, R. D. Chambers, and J. G. Santiago, Lab Chip 9, 2437 (2009).

[49] A. Persat, R. D. Chambers, and J. G. Santiago, [http://microfluidics.stanford.edu/download].

[50] This value follows from Henry's law with the coefficient $c_{\mathrm{CO}_{2(\mathrm{aq})}} / p_{\mathrm{CO}_{2(\mathrm{~g})}}=3.5 \mathrm{mM} /$ atm [51] for $\mathrm{CO}_{2(\mathrm{~g})} \rightleftharpoons \mathrm{CO}_{2(\mathrm{aq})}$ and the reaction constant $K_{\mathrm{H}_{2} \mathrm{CO}_{3}}=2.6 \times 10^{-3}$ for carbonic acid $\mathrm{H}_{2} \mathrm{O}+\mathrm{CO}_{2(\mathrm{aq})} \rightleftharpoons \mathrm{H}_{2} \mathrm{CO}_{3}[48]$.

[51] H. S. Harned and R. Davis, J. Am. Chem. Soc. 65, 2030 (1943).

[52] A. Persat, M. E. Suss, and J. G. Santiago, Lab Chip 9, 2454 (2009).

[53] D. R. Lide, CRC Handbook of Chemistry and Physics, 91st ed. (Internet Version 2011) (CRC/Taylor and Francis, Boca Raton, FL, 2010).

[54] J. Kielland, J. Am. Chem. Soc. 59, 1675 (1937).

[55] D. C. Grahame, Chem. Rev. 41, 441 (1947).

[56] V. G. Levich, Physicochemical Hydrodynamics (Prentice-Hall, New York, 1962).

[57] I. Rubinstein, Physical Electrochemistry (Dekker, New York, 1995).

[58] H. Bruus, Theoretical Microfluidics, Oxford Master Series in Physics No. 18 (Oxford University Press, Oxford, 2008).

[59] M. S. Kilic, M. Z. Bazant, and A. Ajdari, Phys. Rev. E 75, 021502 (2007).

[60] F. Persson, L. H. Thamdrup, M. B. Mikkelsen, S. E. Jarlgaard, P. Skafte-Pedersen, H. Bruus, and A. Kristensen, Nanotechnology 18, 245301 (2007).

[61] K. Huang and R. Yang, Nanotechnology 18, 115701 (2007). 